\title{
Lineage-specific BCL11A knockdown circumvents toxicities and reverses sickle phenotype
}

\author{
Christian Brendel, ${ }^{1,2}$ Swaroopa Guda, ${ }^{1,2}$ Raffaele Renella, ${ }^{1,2,3}$ Daniel E. Bauer, ${ }^{1,2,3}$ Matthew C. Canver, ${ }^{2}$ Young-Jo Kim, ${ }^{2,4}$ \\ Matthew M. Heeney, ${ }^{1,2,3}$ Denise Klatt, ${ }^{5}$ Jonathan Fogel, ${ }^{1}$ Michael D. Milsom, ${ }^{6}$ Stuart H. Orkin, ${ }^{1,2,3,7,8}$ \\ Richard I. Gregory, ${ }^{1,2,8,9}$ and David A. Williams ${ }^{1,2,3,8}$
}

\begin{abstract}
'Division of Hematology/Oncology, Boston Children's Hospital, Boston, Massachusetts, USA. ${ }^{2}$ Harvard Medical School, Boston, Massachusetts, USA. ${ }^{3}$ Department of Pediatric Oncology, Dana-Farber Cancer Institute, Boston, Massachusetts, USA. " Department of Orthopedic Surgery, Boston Children's Hospital, Boston, Massachusetts, USA. ${ }^{5}$ Department of Experimental Hematology, Hannover Medical School, Hannover, Germany. ${ }^{6}$ Division of Stem Cells and Cancer, German Cancer Research Center (DKFZ), Heidelberg Institute for Stem Cell Technology and Experimental Medicine, Heidelberg, Germany. ${ }^{7}$ Howard Hughes Medical Institute, Boston, Massachusetts, USA. ${ }^{~}$ Harvard Stem Cell Institute, Harvard University, Boston, Massachusetts, USA. ${ }^{9}$ Stem Cell Program, Boston Children's Hospital, Department of Biological Chemistry and Molecular Pharmacology, Harvard Medical School, Boston, Massachusetts, USA.
\end{abstract}

\begin{abstract}
Reducing expression of the fetal hemoglobin (HbF) repressor BCL11A leads to a simultaneous increase in $\gamma$-globin expression and reduction in $\beta$-globin expression. Thus, there is interest in targeting BCL11A as a treatment for $\beta$-hemoglobinopathies, including sickle cell disease (SCD) and $\beta$-thalassemia. Here, we found that using optimized shRNAs embedded within an miRNA (shRNA ${ }^{\mathrm{miR}}$ ) architecture to achieve ubiquitous knockdown of BCL11A profoundly impaired long-term engraftment of both human and mouse hematopoietic stem cells (HSCs) despite a reduction in nonspecific cellular toxicities. BCL11A knockdown was associated with a substantial increase in S/C2-phase human HSCs after engraftment into immunodeficient (NSG) mice, a phenotype that is associated with HSC exhaustion. Lineage-specific, shRNA ${ }^{\text {miR }}$-mediated suppression of BCL11A in erythroid cells led to stable long-term engraftment of gene-modified cells. Transduced primary normal or SCD human HSCs expressing the lineage-specific BCL11A shRNAmiR gave rise to erythroid cells with up to $90 \%$ reduction of BCL11A protein. These erythrocytes demonstrated $60 \%-70 \%$ $\gamma$-chain expression (vs. $<10 \%$ for negative control) and a corresponding increase in HbF. Transplantation of genemodified murine HSCs from Berkeley sickle cell mice led to a substantial improvement of sickle-associated hemolytic anemia and reticulocytosis, key pathophysiological biomarkers of SCD. These data form the basis for a clinical trial application for treating sickle cell disease.
\end{abstract}

\section{Introduction}

Induction of fetal hemoglobin ( $\mathrm{HbF}$ ) in both sickle cell disease (SCD) and $\beta$-thalassemia is an extremely promising approach to ameliorate the severity of both diseases (1). However, there has been limited success over the past 3 decades in developing small-molecule $\mathrm{HbF}$ inducers that demonstrate consistent clinical efficacy in these diseases. Recent molecular studies have revealed new regulators of the fetal-to-adult hemoglobin switch in humans, including BCL11A (2-5). BCL11A is an essential transcription factor required for $B$ lymphocyte development $(6,7)$. While $B c l 11 a^{-/}$mice lack B lymphocytes, Xu et al. have demonstrated significant rescue of the hemolytic anemia and end-organ damage of a humanized SCD mouse model crossed onto a mouse background with conditional deletion of Bcl11a in erythroid cells (8). Thus, BCL11A is a genetically and functionally validated regulator of $\gamma$-globin expression and a prime candidate for targeted therapy aimed at induction of $\mathrm{HbF}$ in individuals with SCD.

Authorship note: C. Brendel and S. Guda contributed equally to this work. Conflict of interest: The authors have declared that no conflict of interest exists. Submitted: April 8, 2016; Accepted: July 28, 2016.

Reference information: J Clin Invest. 2016;126(10):3868-3878. doi:10.1172/JCl87885.
Curative treatment for SCD can be attained with hematopoietic stem cell transplantation (HSCT). Using matched related donors, greater than $85 \%$ disease-free survival has been reported (9). Graft failure and transplant-related mortality contribute to the significant complications associated with allogeneic HSCT in SCD. Favorable outcomes in SCD are largely dependent on the availability of matched sibling donors and the incidence of graft failure and graft versus host disease (GVHD). Fewer than 10\% of SCD patients have unaffected HLA-matched sibling potential donors (10). In a published series of SCD patients treated with HSCT, there was $~ 20 \%-25 \%$ risk of serious GVHD and $10 \%$ risk of chronic GVHD, which contributes to late mortality (11).

Gene therapy for the hemoglobinopathies offers the clear advantage of eliminating the risk of GVHD and the need to identify suitable stem cell donors by the use of autologous cells. Gene therapy trials are being developed or are underway to express either $\mathrm{HbF}$ or sickling-resistant $\mathrm{HbA}$ variants (12-15). However, targeting BCL11A in SCD holds the significant advantage that adequate knockdown of BCL11A in erythroid cells derived from gene-modified hematopoietic stem cells (HSCs) will increase $\mathrm{HbF}$ expression while concurrently reducing expression of the sickle hemoglobin (HbS) mutant. Since hemoglobin polymerization in sickle RBCs is highly dependent on the intracellular concentration of $\mathrm{HbS}$ and is strongly inhibited by 
A

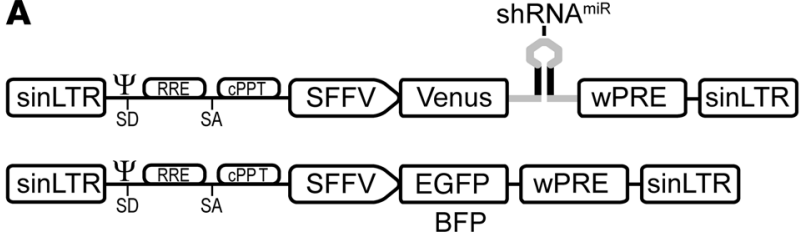

B

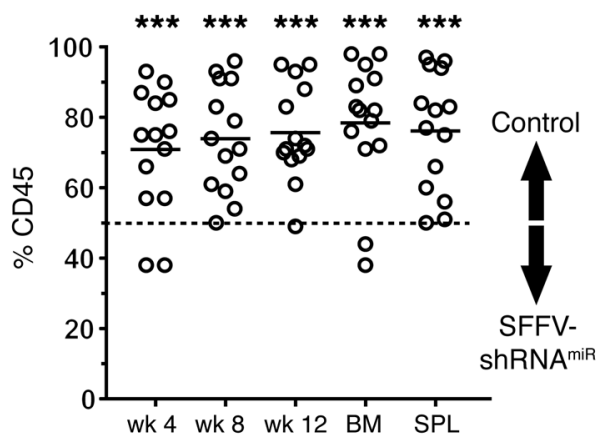

D

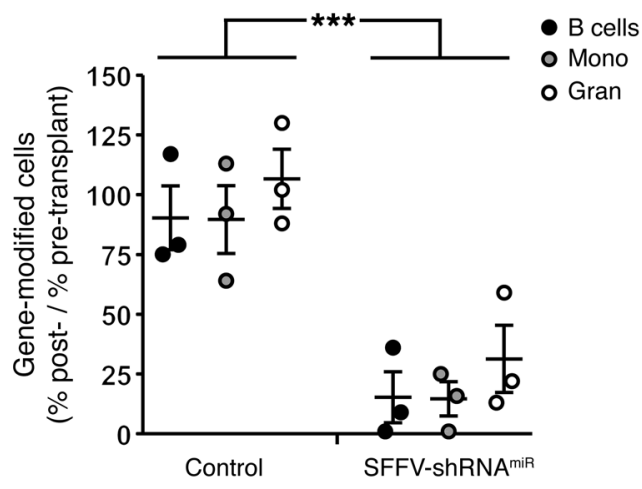

C
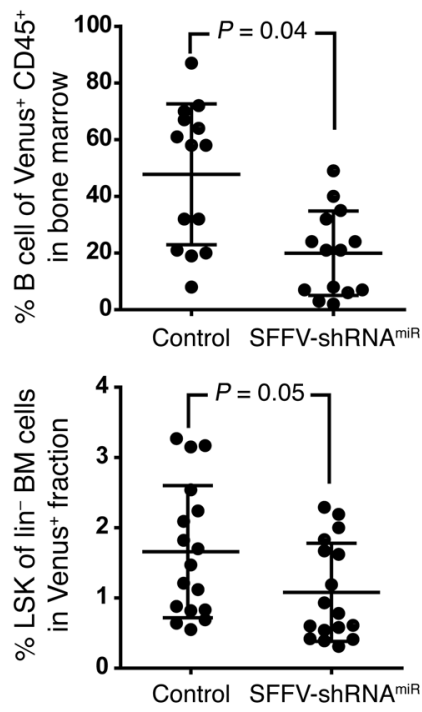

E

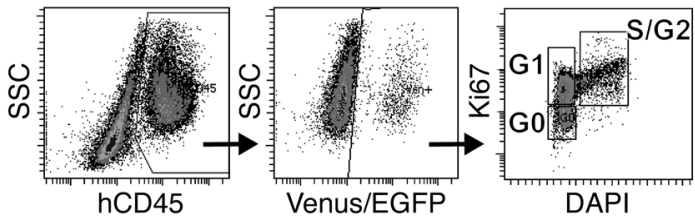

F

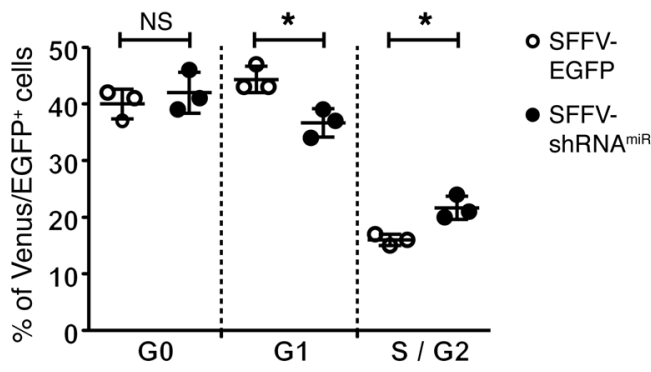

Figure 1. Knockdown of BCL11A reduces engraftment of murine and human hematopoietic stem cells. (A) Lentiviral vectors used in competitive transplantation assays: the SFFV-shRNA ${ }^{\text {miR }}$ vector is shown above and the control vectors are shown below. The dark vertical lines represent the shRNA targeting BCL11A. (B) Murine competitive repopulation assays. The contribution of gene-modified cells in repopulated mice was analyzed in peripheral blood (PB), bone marrow (BM), and spleen (Spl) (week 12). The dashed line indicates the predicted hematopoietic output based on the input of cells. Data points above or below this line indicate an overrepresentation of gene-modified cells derived from the control vector (above the line) or the SFFV-shRNA ${ }^{\text {miR }}$ transduced population (below the line). (C) Reconstituted murine bone marrow B cell compartment (left panel) derived from HSCs transduced with SFFV-shRNA ${ }^{\text {miR }}$ versus control vectors and the LSK content (right panel). Each data point in $\mathbf{B}$ and $\mathbf{C}$ represents an individual recipient. $P$ values are indicated in the figure. (D) Reconstitution of NSG mice with transduced human cells. Human CD34 ${ }^{+}$cells were transduced with SFFV-shRNA ${ }^{\text {miR }}$ or SFFV-EGFP and transplanted into NSG mice. The percentage of gene-modified human B cells, monocytes, and granulocyte fractions at 14 weeks relative to the initial transduction rate is shown. (E) NSG mice were transplanted with SFFV-shRNA ${ }^{\text {miR }}$ - or SFFV-EGFP-transduced hCD34+ cells. The cell cycle status of human gene-modified cells in the bone marrow at 2 weeks was assessed by DNA/Ki76 staining. Representative flow diagrams are shown. (F) The cell cycle distribution of gene-modified cells in vivo (as in panel E) was quantified. Each data point represents an individual mouse. ${ }^{* *} P<0.001 ;{ }^{*} P<0.05$. Error bars in all figures = SD. Statistical analysis: 2 -tailed $t$ test. $\Psi$, packaging signal; cPPT, central polypurine tract; Gran, granulocytes; Mono, monocytes; NS, not significant; RRE, rev-responsive element; SA, splice acceptor; SD, splice donor; SFFV, spleen focus-forming virus promoter; sinLTR, self-inactivating long terminal repeat; SSC, side scatter; wPRE, woodchuck hepatitis virus posttranscriptional regulatory element.

HbF, vectors effectively targeting BCL11A should prevent the cellular phenotype of HbS-containing RBCs. Reduced hemoglobin polymerization would thus lead to a pronounced increase in the RBC half-life in vivo (16). Gene transfer technologies have been established in proof-of-principle human trials as therapeutic options for life-threatening monogenic diseases (reviewed in ref. 17). These successes and the low genotoxicity of lentiviral vectors broaden the spectrum of indications for which gene therapy represents a treatment option (18).

Downregulation of BCL11A expression by small hairpin RNAs (shRNAs) expressed by polymerase (pol) III promoters in lentivirus vectors leads to rapid and sustained reactivation of $\gamma$-globin 
A

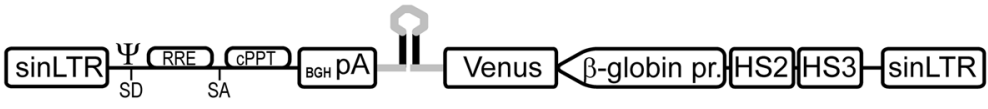

B

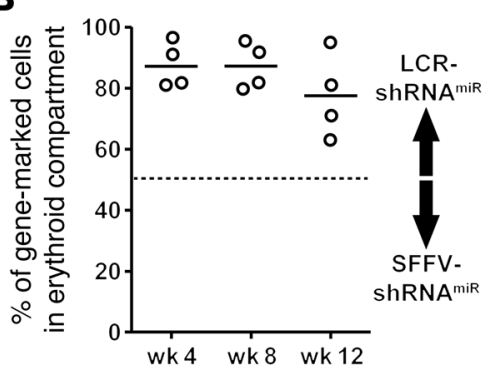

D
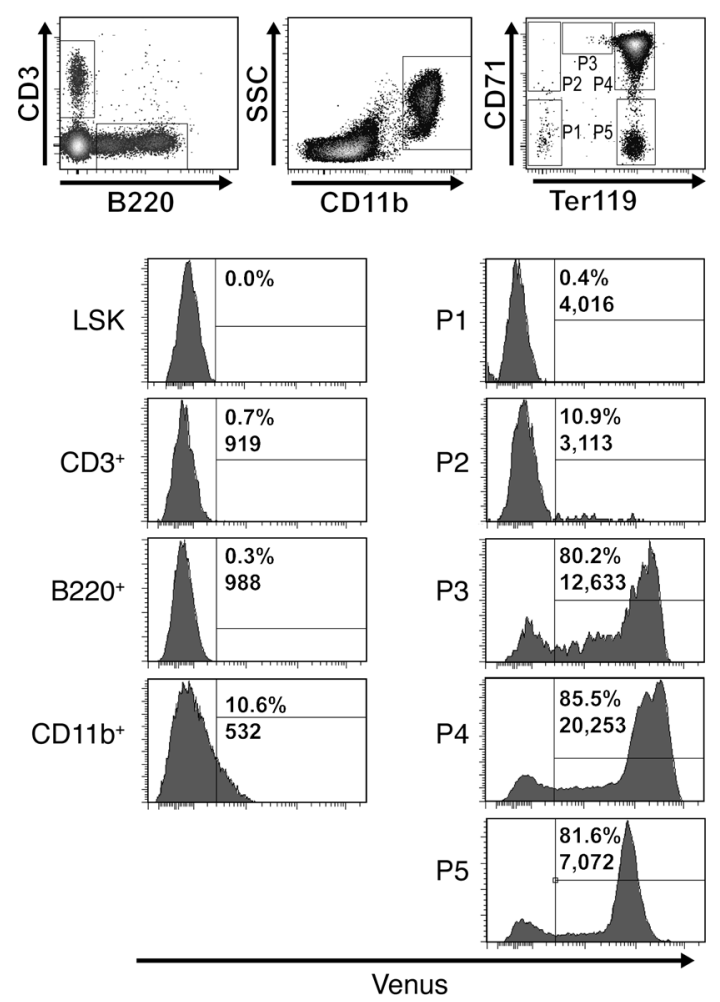

C

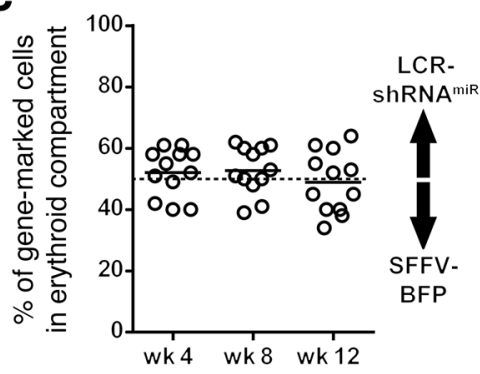

E

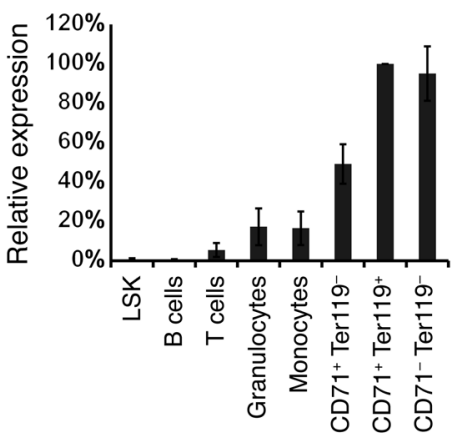

Figure 2. Erythroid-specific expression of the shRNA ${ }^{\text {miR }}$ prevents engraftment defect of hematopoietic stem cells. (A) Configuration of the erythroid-specific LCR-shRNA ${ }^{\text {miR }}$ vector. (B) Competitive repopulation assay between LCR-shRNA ${ }^{\text {mir }}$ - and SFFV-shRNA ${ }^{\text {mir }}$-transduced cells. The relative contribution of gene-modified cells in peripheral blood erythroid cells (defined by CD71 and Ter119 positivity) of transplanted animals was analyzed at 4, 8 , and 12 weeks after transplantation. The dashed line indicates the predicted balanced hematopoietic output. Data points above or below this line indicate an overrepresentation of gene-modified cells derived from the LCR vector- (above the line) or the SFFV-shRNA ${ }^{\text {miR }}$-transduced (below the line) population. Each data point represents an individual recipient mouse. (C) Competitive repopulation between LCR-shRNA ${ }^{\text {miR }}$ - and SFFV-BFP- (empty vector) transduced cells. Analysis as described in panel B. (D) In vivo expression profile of the LCR-shRNA ${ }^{\text {miR }}$ vector in mice. The gating strategy is shown at the top and expression of Venus in different cell lineages is shown below. Numbers in plots indicate percentage Venus ${ }^{+}$cells and the mean fluorescence intensity. (E) Summary of the data shown in $\mathbf{D}$ normalized to the percentage Venus ${ }^{+}$cells in the CD71+Ter119+ population. Data are the mean \pm SD, $n=3$. HS2 and HS3, DNAse hypersensitive sites 2 and 3 from the $\beta$-globin locus control region; LCR, $\beta$-globin locus control region and $\beta$-globin proximal promoter; SFFV, spleen focus-forming virus promoter; SSC, side scatter.

expression and induction of $\mathrm{HbF}\left(\alpha_{2} \gamma_{2}\right)$ expression in adult erythroid precursor cells (5). However, high-level expression of shRNAs in mammalian cells typically using pol III promoters can be associated with nonspecific cellular toxicities, including increased mortality in mice in some experimental transgenic model systems $(19,20)$. Indeed, we have recently shown that pol II-driven
microRNA-adapted shRNAs (shRNA ${ }^{\text {miR) }}$ targeting BCL11A led to significantly increased target knockdown while avoiding nonsequence-specific cytotoxicity associated with pol III promoterdriven shRNAs (21). Here we show that knockdown of BCL11A unexpectedly and profoundly impairs long-term engraftment of both human and mouse HSCs in a sequence-specific fashion. 
We demonstrate that use of erythroid-specific expression of shR$\mathrm{NA}^{\mathrm{miR}}$ targeting BCL11A both circumvents this toxicity and effectively induces $\mathrm{HbF}$ in human erythroid cells, largely attenuating the hematologic effects of SCD.

\section{Results}

Ubiquitous knockdown of BCL11A in hematopoietic stem and progenitor cells impairs hematopoietic reconstitution after transplantation. We previously identified highly effective shRNAs targeting the BCL11A mRNA (21). These shRNAs, targeting sequences of the mRNA that are conserved between mice and humans, were subsequently engineered into pol II-driven shRNA ${ }^{\mathrm{miR}} \mathrm{s}$ in lentivirus vectors to reduce nonspecific toxicities related to shRNA overexpression (21-23). In order to attain high-efficiency knockdown of BCL11A in hematopoietic cells, the shRNA ${ }^{\mathrm{miR}}$ was expressed from a strong SFFV promoter (Figure 1A) mediating ubiquitous expression. Murine hematopoietic stem and progenitor cells (HSPCs) transduced with these lentivirus vectors were utilized for in vivo hematopoietic repopulation experiments. Surprisingly, in pilot experiments we noted a consistent loss of gene-modified cells in vivo that occurred after initial engraftment (data not shown).

To investigate this apparent engraftment phenotype more carefully, we utilized in vivo competitive repopulation experiments for quantitative HSC phenotype analysis. Lineage-negative bone marrow cells isolated from BoyJ (CD45.1) and $\mathrm{Bl} / 6$ mice (CD45.2) were transduced ex vivo with a vector expressing a shRNA ${ }^{\text {miR }}$ against BCL11A or a control vector and mixed to obtain equal fractions of gene-modified cells for engraftment. The cells were subsequently transplanted into congenic CD45.1/CD45.2 mice, allowing for identification of both donor populations and residual host cells. To validate the specificity of BCL11 knockdown in any toxicity, 3 different shRNA ${ }^{\text {miR }}$ s targeting BCL11A were tested. As controls, a nontargeting shRNA ${ }^{\mathrm{miR}}(\mathrm{NT})$ and a vector expressing only a blue fluorescent protein (BFP) reporter also driven by the SFFV promoter (SFFV-BFP) were used (Figure 1A). The contribution of gene-modified cells in transplanted animals was determined in peripheral blood, bone marrow, and spleen at 4, 8, and 12 weeks after transplantation (Figure 1B). Minor differences in the ratio of the infused transduced cells were corrected in the analysis.

For the purpose of statistical analysis and because they yielded comparable results, data from all 3 BCL11A-targeting shRNA ${ }^{\text {mir }}$ s and both control vectors were combined in each grouping and are shown in Figure 1. Confirming the data from the pilot engraftment studies, cells transduced with vectors targeting BCL11A were outcompeted by cells transduced with control vectors, indicating a selective disadvantage due to BCL11A knockdown. Since BCL11A has previously been shown to be a critical transcription factor for $\mathrm{B}$ cell development, we performed a more detailed analysis of the B lymphocyte compartment. Analysis of B cells derived from transduced HSCs demonstrated a significantly reduced fraction of B220-positive B cells upon BCL11A knockdown (Figure 1C), validating lineage-specific toxicity of these vectors. Since the engraftment of all hematopoietic lineages was affected (data not shown), we next studied the HSPC compartment in these mice. This analysis confirmed a significant loss of more primitive lin', $\mathrm{Sca}_{-1}{ }^{+}, \mathrm{c}-\mathrm{Kit}^{+}$(LSK) cells that include the engrafting HSC compartment (Figure 1C). To determine if the adverse effect of BCL11A knockdown in HSPC was species specific, transduced human granulocyte colony-stimulating factor-mobilized (G-CSF-mobilized) peripheral blood $\mathrm{CD}^{2} 4^{+}$cells transduced with SFFV-shRNA ${ }^{\mathrm{miR}}$ or the SFFV-EGFP vector were transplanted into nonobese diabetic/ severe combined immunodeficient and IL-2 receptor $\gamma$ subunitdeficient (NSG) immunodeficient mice. Analysis of bone marrow cells was performed 14 weeks later and the fraction of gene-modified cells recovered from bone marrow after engraftment was compared with the fraction of gene-modified cells present in the initially transplanted cells (Figure 1D). A significant loss of gene-modified cells derived from the SFFV-shRNA ${ }^{\text {miR_-transduced }}$ $\mathrm{CD} 4^{+}$cells compared with controls was observed, confirming the effect of BCL11A knockdown in human HSPCs.

Next, in order to determine the potential mechanism of reduced engraftment due to BCL11A knockdown, we performed progenitor colony-forming assays in transduced murine cells and apoptosis and cell cycle assays on human $\mathrm{CD} 34^{+}$cells in vitro. The experimental group was transduced with SFFV-shRNA ${ }^{\text {miR }}$ vectors targeting BCL11A, while the control groups were again transduced with SFFV-shRNA ${ }^{\text {miR NT }}$ or SFFV-EGFP/BFP vectors. No differences could be detected in the in vitro growth (Supplemental Figure 1A; supplemental material available online with this article; doi:10.1172/JCI87885DS1) and composition (Supplemental Figure $1, \mathrm{~B}$ and $\mathrm{C}$ ) of murine progenitor colonies after transduction with BCL11A knockdown vector. Consistent with this apparent lack of toxicity in murine cells, apoptosis and cell cycle analysis showed no differences between the groups in transduced human CD34derived cells (Supplemental Figure 2, A and B). These data suggest a specific phenotype during or after in vivo engraftment and reconstitution that was not apparent in in vitro cultured cells. We thus transplanted CD $34^{+}$cells transduced with SFFV-shRNA ${ }^{\text {miR }}$ vectors targeting BCL11A into NSG mice and analyzed the presence and cell cycle status of the gene-modified cells at an early time point after infusion (Figure 1E). We found a reduced number of transduced cells in G1 and an increased number of cells in the S/G2 phase (Figure $1 F)$. This suggests that BCL11A knockdown compromises cell cycle regulation within the HSC compartment at the G1/S transition, resulting in an elevated proliferative index and compromised functional activity. This is consistent with a recent report of increased cell cycling in murine Bcl11 $a^{-/}$HSC (24) and indicates a conserved but not fully defined role for BCL11A in HSCs across species.

Erythroid-specific knockdown of BCL11A circumvents toxicities in engrafting HSCs. The negative impact of BCL11A knockdown on engraftment seen in gene-modified HSCs makes gene therapy using constitutively active promoters appear unfeasible. We hypothesized that erythroid-specific knockdown of BCL11A could circumvent the adverse effects on HSCs and B lymphocytes while maintaining the therapeutic effect of $\gamma$-globin induction in erythroid cells. The success of this approach would depend upon tight regulation of expression in only erythroid cells and induction of expression at a stage of RBC development that effects appropriate modulation of BCL11A and induction of $\gamma$-globin. To test this hypothesis, we generated a lentivirus vector incorporating the BCL11A shRNA ${ }^{\text {miR }}$ cassette and the Venus fluorescent reporter under transcriptional control of the minimal $\beta$-globin proximal promoter linked to hypersensitive sites 2 and 3 (HS2 and HS3) of the $\beta$-globin locus control region (LCR; see ref. 25) 
A

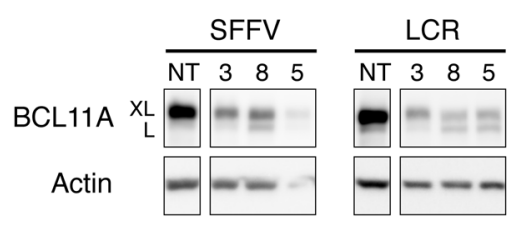

D
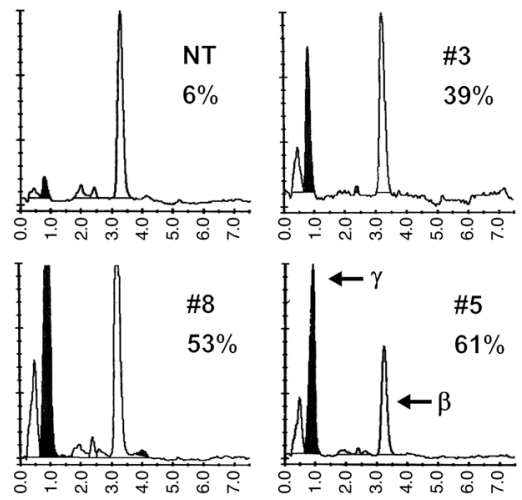

B - SFFV-ShRNAmir

LCR-ShRNA

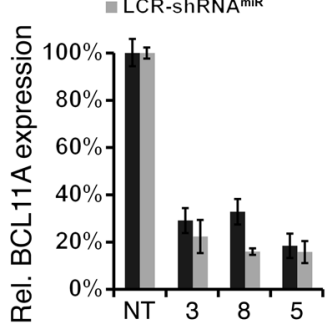

$\mathbf{E}$

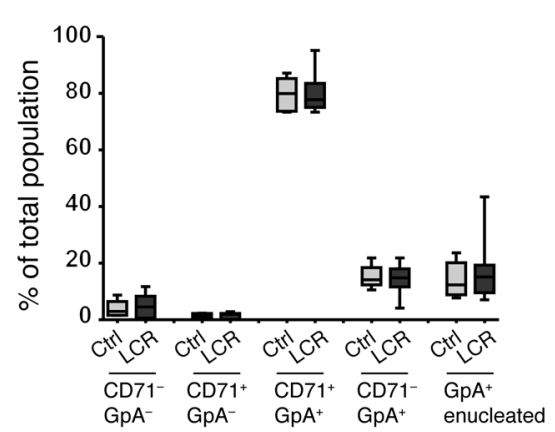

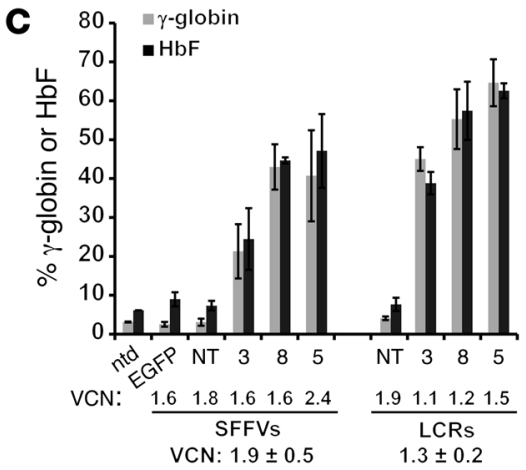

$\mathbf{F}$

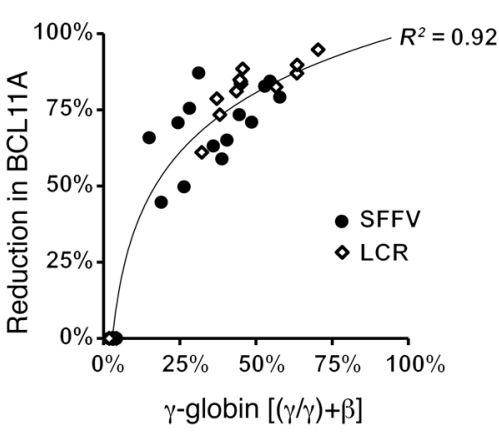

Figure 3. Efficient knockdown of BCL11A leads to high $\gamma$-globin and HBF induction in human primary cells in vitro. (A) Representative Western blot showing a side by side comparison of BCL11A knockdown efficiency mediated by SFFV or LCR-shRNA ${ }^{\text {miR }}$ vectors in erythroid cells differentiated in vitro from transduced hCD34+ cells. The top panels show BCL11A immunoblot (XL and L isoforms) and the bottom panels show actin as loading control. Different lanes represent different shRNA targeting sequences (see methods). (B) Quantification of Western blots derived from 3 independent experiments. Data represent the mean \pm SD of densitometric analysis of immunoblots. (C) Induction of $\gamma$-globin (by RT-qPCR) and HbF induction (by HPLC) of erythroid cells differentiated in vitro from transduced hCD34+ cells. Data are the mean $\pm S D, n=3$. Vertical arrows indicate the shRNA ${ }^{\text {miR }} s$ chosen for further experiments. Average vector copy numbers (VCNs) are indicated below. (D) Representative HPLC plots showing HbF (black) and HbA (white) peaks for the 3 selected candidates. (E) Differentiation state of controls (mean of NT and SFFV-EGFP) and LCR-shRNA ${ }^{\text {miR }}$ (mean of 3, 5, and 8) vector-transduced CD34 cells after 18 days of erythroid culture. $n=3$. (F) Correlation between the degree of knockdown of BCL11A and induction of HbF in transduced hCD34+ cells after erythroid in vitro differentiation. Each data point represents an individual sample. LCR, $\beta$-globin locus control region and $\beta$-globin proximal promoter; NT, nontargeting shRNA ${ }^{\text {mir; }}$; ntd, nontransduced; SFFV, spleen focus-forming virus promoter.

(LCR-shRNA ${ }^{\text {miR }}$, Figure 2A). To demonstrate the lack of a negative effect on HSC engraftment, the impact of the LCR vector versus the SFFV vector was first assessed in competitive repopulation experiments. Mice were repopulated with lineage-negative cells transduced with LCR-shRNA ${ }^{\text {miR }}$ expressing Venus or SFFV-shRNA $^{\text {miR }}$ vector expressing Tomato. As the LCR vector is transcriptionally silent in lin- cells, an aliquot of cells used for transplantation was subjected to in vitro erythroid differentiation and the ratio of Venus ${ }^{+}$cells measured in the transcriptionally permissive $\mathrm{CD}^{+} 1^{+} \mathrm{Ter} 119^{+}$population was used for normalization of the ratio of $\mathrm{SFFV}^{-}$versus LCR-transduced cells. Fluorescent reporter expression in erythroid cells of transplanted animals was compared at various time points during erythroid differentiation. As seen in Figure 2B, reconstitution of the erythroid compartment was uniformly superior in the LCR-transduced group compared with the SFFV-transduced group. Although this suggests lower HSC toxicity of the LCR-shRNA ${ }^{\text {miR }}$ vector compared with the SFFV-sh$\mathrm{RNA}^{\mathrm{miR}}$ vector, in order to confirm this lack of toxicity in engrafting HSCs due the presence of the shRNA ${ }^{\text {mir }}$, we directly compared engraftment of LCR-shRNA ${ }^{\text {miR }}$-transduced HSCs with a neutral vector (SFFV-BFP). As seen in Figure 2C, reconstitution in vivo was balanced, with hematopoietic cells derived from each vector.
Also, no differences between treatment groups were observed in the frequency of B cells, which have previously been shown to require BCL11A expression for development (data not shown). Furthermore, in initial pilot studies, LCR-shRNA ${ }^{\text {miR }}$-transduced cells were serially transplanted into secondary animals with a total observation time of 24 weeks ( 12 weeks +12 weeks). Three of three secondary animals engrafted and gene-marked erythrocytes were produced (data not shown). The lack of HSC toxicity appears to be a result of the high fidelity of erythroid-specific expression mediated by LCR-promoter/enhancer transcriptional regulatory elements (Figure 2, D and E). Even at a high multiplicity of infection, where transduction rates of greater than $80 \%$ were observed in erythrocytes, the vector remained transcriptionally silent in $\mathrm{T}, \mathrm{B}$, and LSK cells, with weak expression found only in CD11 $\mathrm{b}^{+}$ myeloid cells (Figure 2D). In contrast, expression was continuously upregulated during erythroid differentiation, beginning in the CD71+Ter119- stage of differentiation, with peak expression levels found in more mature $\mathrm{CD} 71^{+} \mathrm{Te} 119^{+}$erythroid precursors (Figure 2, D and E). In CD71+Ter $119^{+}$cells, the mean fluorescence intensity (MFI) exceeded the levels observed with SFFV-mediated expression vectors when vector copy numbers were equivalent (data not shown). In fully mature CD $71^{-}{ }^{-}{ }^{-1119^{+}}$erythrocytes the 
A

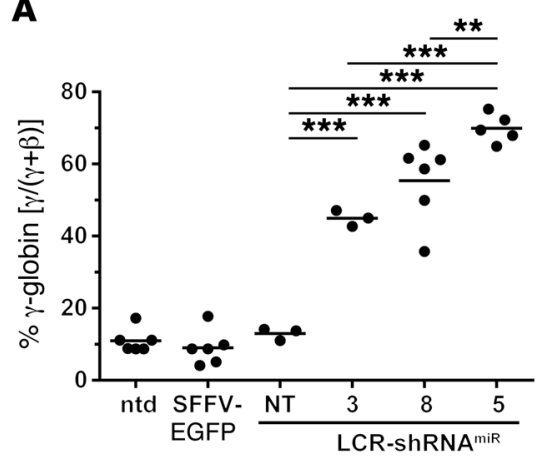

C

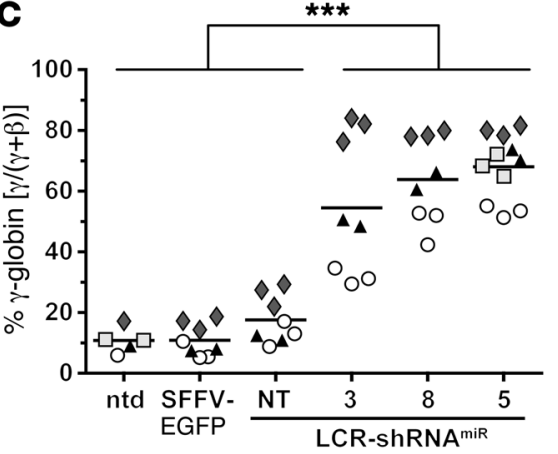

B
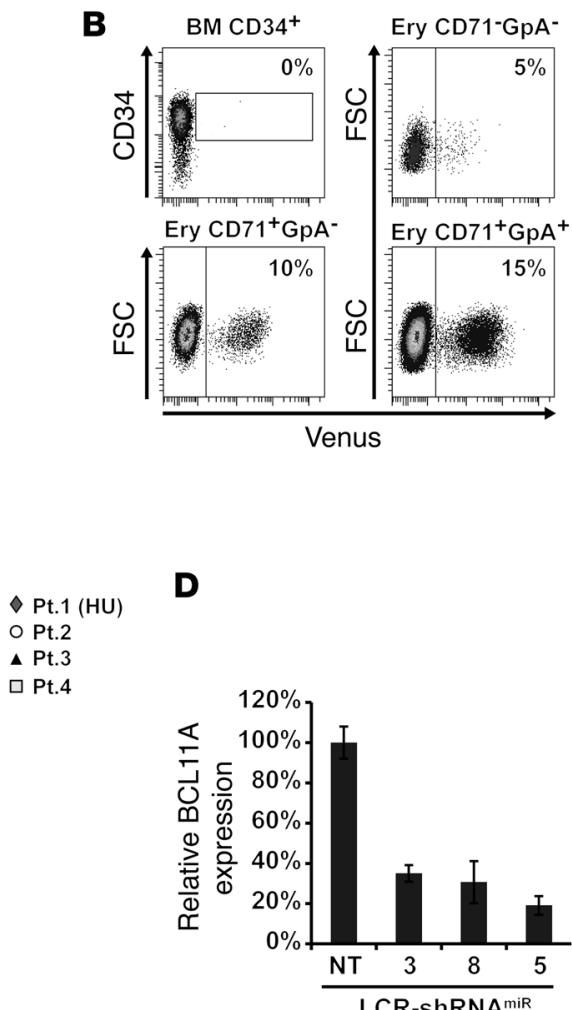

D

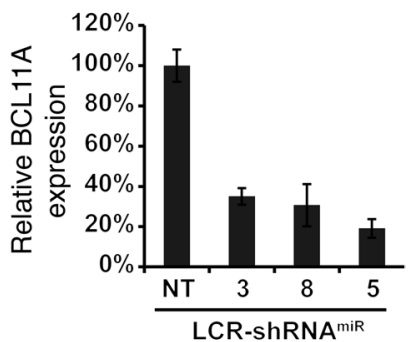

Figure 4. The LCR-shRNA ${ }^{\text {miR }}$ vector does not inhibit human hematopoietic stem cell engraftment in NSG mice and induces $\gamma$-globin in erythroid cells derived from engrafted normal and SCD patient CD34+ cells. (A) Healthy donor-derived CD34+ cells were transduced and transplanted into NSG-mice. Fourteen to 16 weeks later, $\mathrm{CD}_{4} 4^{+}$cells were isolated from the bone marrow and subjected to erythroid in vitro differentiation followed by FACS sorting and RT-qPCR analysis. Each data point represents the result from an individual animal. (B) Specificity of the LCR-shRNA ${ }^{\text {miR }}$ vector in human cells isolated from the bone marrow (BM) of NSG mice. Shown is the percentage of Venus ${ }^{+}$cells in freshly isolated CD34+ cells and in 3 different erythroid (Ery) maturation stages 1 week after start of erythroid in vitro differentiation. (C) Sickle cell patient CD34+ cells were transduced and differentiated in vitro. RT-qPCR analysis was performed on FACS sorted transduced cells after 18 days of culture. Symbols indicate different patients and each data point represents an independent replicate. (D) Western blot quantification of BCL11A in transduced erythroid cells derived from a single SCD patient. Data are the mean $\pm \mathrm{SD}, n=3$. ${ }^{* *} P \leq 0.01$; ${ }^{* *} P \leq 0.001$. Statistics: 1 -way ANOVA. FSC, forward scatter; HU, hydroxyurea; LCR, $\beta$-globin locus control region and $\beta$-globin proximal promoter; NT, nontargeting shRNA ${ }^{\text {miR; }}$, ntd, nontransduced; SFFV, spleen focus-forming virus promoter.

MFI was reduced, presumably as a consequence of enucleation and the erythrocyte half-life exceeding the fluorophore half-life.

LCR vector-mediated erythroid-specific knockdown of BCL11A yields a high level of induction of $\gamma$-globin and $\mathrm{HbF}$ in human erythroid cells in vitro. The precise stage during erythroid maturation at which BCL11A expression is required to repress the $\gamma$-globin locus is not known. To determine if the timing of LCR-mediated shRNA ${ }^{\text {miR }}$ expression effectively derepressed $\gamma$-globin and induced $\mathrm{HbF}$, we analyzed erythroid cells differentiated in vitro from transduced human G-CSF-mobilized CD34 ${ }^{+}$HSPCs (Supplemental Figure 3A). The cells were transduced with the LCR or SFFV vector expressing an NT control and the 3 most potent shRNA ${ }^{\text {miR }}$ s (shRNA ${ }^{\text {miR }} 3,-5$, and -8) as defined by previous in vitro studies with SFFV-shRNA ${ }^{\text {miR }}(21)$. BCL11A protein expression was determined by immunoblot after 11 days of erythroid differentiation in Venus-positive cells; $\gamma$-globin mRNA and HbF levels were analyzed on day 18. As seen in Figure 3A, both LCR and SFFV vectors mediated substantial knockdown of BCL11A relative to the NT control. Densitometric quantification showed that LCR-shRNA ${ }^{\text {miR }}$ vectors led to equivalent or slightly higher BCL11A knockdown compared with SFFV-shRNA ${ }^{\text {mir }}$ vectors. The most effective vector (LCR-shRNA ${ }^{\text {miR }}$ ) mediated greater than $80 \%$ knockdown of BCL11A (Figure 3B). Improved knockdown efficiencies were associated with higher levels of $\gamma$-globin and $\mathrm{HbF}$ induction, with LCR-shRNA ${ }^{\text {miR }} 5$ leading to $~ 60 \% \mathrm{HbF}$ (vs. $\sim 10 \%$ in NT control) in erythroid cells (Figure 3, C and D). When comparing the results of shRNA ${ }^{\text {miR }} 3,-5$, and -8, LCR-shRNA ${ }^{\text {miR }}$ vectors were efficient in $\mathrm{HbF}$ induction at equivalent or slightly lower average vector copy numbers compared with the SFFV group (1.3 \pm 0.2 vs. $1.9 \pm 0.5$, respectively).

To examine the effects of BCL11A knockdown on RBC development, erythroid differentiation of transduced $\mathrm{CD} 34^{+}$cells during in vitro culture was monitored using CD71 and GpA surface markers and DAPI DNA stain for enucleation (Supplemental Figure 3B). There were no alterations in erythroid differentiation due to BCL11A knockdown (Figure 3E). To determine if $\gamma$-globin mRNA could be used as a surrogate for BCL11A knockdown and $\mathrm{HbF}$ induction in subsequent experiments, we next correlated $\gamma$-globin mRNA (quantitative reverse transcription PCR [RT-qP$\mathrm{CR}]$ ), $\mathrm{HbF}$ (high-performance liquid chromatography [HPLC]), and BCL11A immunoblot data. $\gamma$-globin mRNA and HbF levels were highly correlated in differentiated cells, allowing for the extrapolation of $\mathrm{HbF}$ based on $\gamma$-globin RT-qPCR in this exper- 

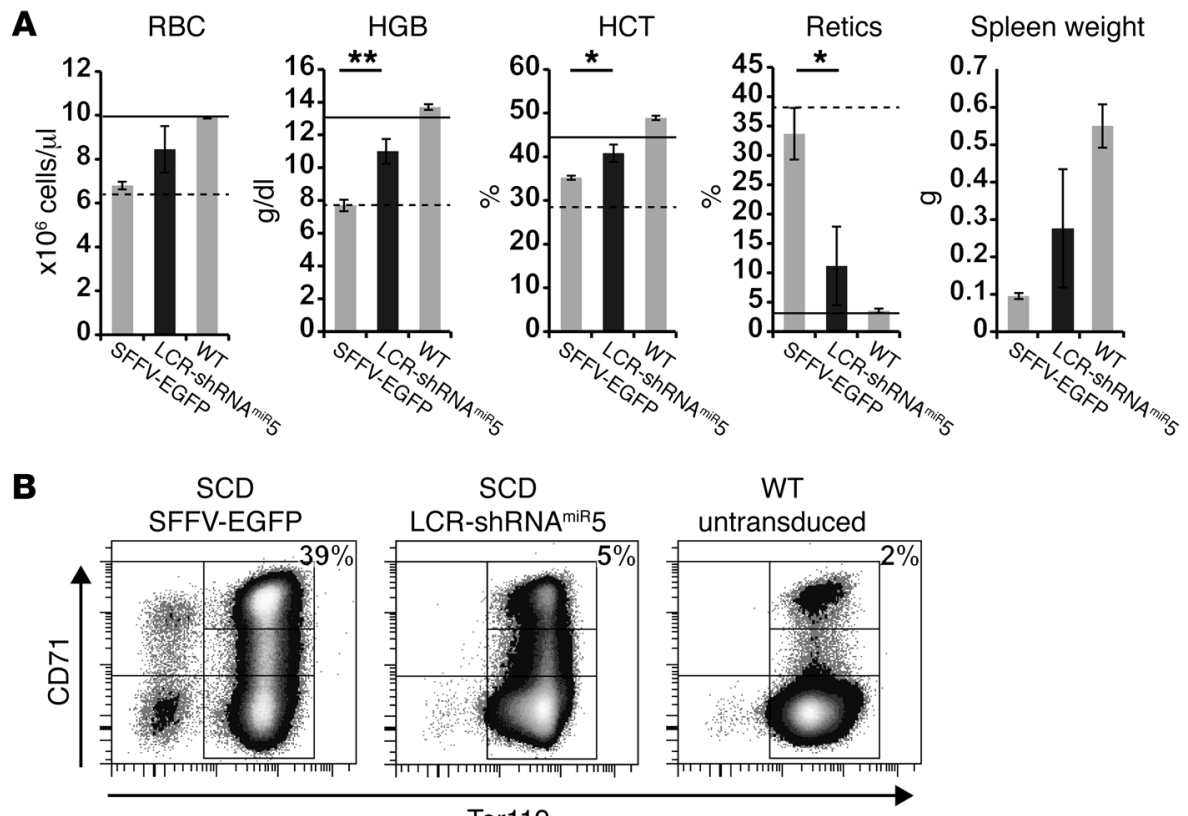

Ter119

Figure 5. Amelioration of SCD-specific disease parameters in vivo using the Berkeley-SCD mouse model. (A) Lineage-negative bone marrow cells from Berkeley-SCD mice were transduced with the LCR-shRNA miR5 vector and transplanted into lethally irradiated Boyj recipients. As controls, SFFV-EGFP-transduced SCD or untransduced WT cells were transplanted and peripheral blood hematologic parameters of transplanted animals were obtained. Published reference values in the nontransplant setting are indicated by lines (dotted line, Berkeley-SCD; continuous line, WT mice). Control groups are shown in light gray, the LCR-shRNA ${ }^{\text {miR }} 5$-transplanted group in dark gray. ${ }^{*} P<0.05 ;{ }^{* *} P<0.01$. (B) Representative FACS plots of peripheral blood cells stained for the erythroid differentiation markers CD71 and Ter119. Percentages in plots indicate the fraction of CD71+Ter119+ high erythroid precursor cells. Data are the mean $\pm \mathrm{SD}, n=3$. HCT, hematocrit; HCB, hemoglobin; LCR, $\beta$-globin locus control region and $\beta$-globin proximal promoter; Retics, reticulocytes; SFFV, spleen focus-forming virus promoter.

imental setting (Supplemental Figure 4A). Furthermore, a nonlinear correlation of BCL11A versus $\gamma$-globin levels (Figure 3F) showed that $50 \%$ reduction in BCL11A was associated with induction of $\mathrm{HbF}$ to $\sim 20 \%$. Similar levels of $\mathrm{HbF}$ have been recently reported for BCL11A haploinsufficient patients $(26,27)$, supporting the applicability and the predictive value of our approach.

LCR-shRNA ${ }^{\text {miR }}$ gene-modified cells engraft NSG mice and lead to high levels of $\gamma$-globin induction in sickle RBCs. We next determined if erythroid cells derived from engrafted human transduced CD $34^{+}$ cells would show induction of $\mathrm{HbF}$. Healthy donor $\mathrm{CD} 34^{+}$cells transduced with LCR-shRNA ${ }^{\text {miR }} 3,-5$, and -8 (Figure 3 ) were transplanted into NSG mice. LCR-shRNA ${ }^{\text {miR }} \mathrm{NT}$ - or SFFV-EGFP-transduced cells and untransduced $\mathrm{CD} 34^{+}$cells served as controls. Engraftment and lineage distribution were similar between treatment groups (Supplemental Figure 5, A and B). As human erythropoiesis is poorly modeled in NSG mice, human $\mathrm{CD}_{3} 4^{+}$cells were harvested from the bone marrow of transplanted animals 14-16 weeks after transplant and differentiated in vitro into erythroid cells. RT-qPCR analyses for $\gamma$-globin content in Venus-positive cells revealed means of $\sim 45 \%, \sim 55 \%$, and $\sim 68 \% \gamma$-globin for shRNA ${ }^{\text {miR }} 3,-8$ and -5 , respectively (Figure 4A). Consistent with the expression pattern in murine cells, Venus was undetectable in freshly isolated engrafted CD $34^{+}$ cells harvested from the bone marrow of NSG mice with increasing expression during erythroid differentiation (Figure 4B).

We next analyzed the performance of LCR-shRNA ${ }^{\text {miR }}$ vectors in bone marrow cells from SCD patients. Bone marrow $\mathrm{CD} 34^{+}$cells from 4 different SCD donors were utilized for transduction with

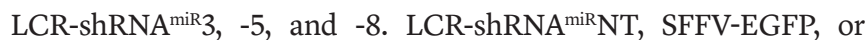
untransduced cells served as controls and subsequent in vitro differentiation. The mean $\gamma$-globin induction level was $54 \%, 62 \%$, and $68 \%$ for shRNA ${ }^{\text {miR }} 3,-8$, and -5 , respectively (Figure $4 \mathrm{C}$ ). Interpatient variation was higher using sickle cell patient-derived $\mathrm{CD} 4^{+}$cells compared with healthy donors, and 1 patient on hydroxyurea (HU) had higher baseline $\gamma$-globin levels. This observation most likely represents intrinsic biological variation rather than technical variation, as replicates from the same donor are closely clustered. Sufficient cells were available from 1 patient sample to perform Western blot, which confirmed that BCL11A levels ranged from $19 \%$ to $36 \%$ relative to cells transduced with shRNA ${ }^{\text {miR }} \mathrm{NT}$ (Figure 4D).

Genetic modification of Berkeley SCD HSCs with LCR-shRNA ${ }^{\text {miR }} 5$ leads to improvement of disease-associated hematological parameters. The LCR-shRNA ${ }^{\text {miR }} 5$ vector was selected as the most promising candidate vector for translation into the clinic due to its superior knockdown of BCL11A and high levels of $\gamma$-globin and HbF induction in all previous experiments. We next determined if characteristic disease parameters could be ameliorated by this vector in a transplantation model utilizing Berkeley SCD (BERK-SCD) mouse HSCs. Lineage-negative bone marrow cells from BERK-SCD mice transduced with LCR-shRNA ${ }^{\text {miR } 5}$ or the SFFV-EGFP (as control) were transplanted into lethally irradiated BoyJ recipient animals. A second control group received untransduced cells isolated from BL/6 animals. Transplantation into BoyJ mice was chosen to circumvent the increased mortality of BERK-SCD upon conditioning and to allow for engraftment analysis using CD45 isotypes. 
Similarly to BERK-SCD mice, BoyJ mice engrafted with BERKSCD develop a severe sickle cell phenotype, with evidence of hemolytic anemia characterized by low hemoglobin and reticulocytosis. These animals were sacrificed 10 weeks after transplantation without prior intermediate analyses because of increased sensitivity to blood drawing procedures and anesthesia. After transplantation, engraftment as measured by the number of CD $45^{+}$cells in peripheral blood was $80 \%$ and similar in both recipient groups receiving BERK-SCD HSCs. The gene marking in Ter $119^{+}$erythroid cells was $\sim 50 \%$ in the SFFV-EGFP and $20 \%$ in the LCR-shRNA ${ }^{\text {miR }} 5$ group (Supplemental Figure 6), reflecting the differences in initial transduction rates related to different titers of vector supernatants.

Engraftment of SFFV-EGFP-transduced HSCs derived from BERK-SCD mice was associated with significantly lower RBC, hemoglobin, and hematocrit levels with increased reticulocytes and spleen weights, indicative of severe hemolytic anemia (Figure 5A). These values mirrored the hematological values of BERK-SCD in a nontransplant setting, supporting the validity of the experimental model. Mice transplanted with HSCs derived from BERK-SCD mice and transduced with the LCR-shRNA ${ }^{\text {miR } 5}$ vector showed improvements in all blood parameters, even at a transduction efficiency of $20 \%$, as determined by the surrogate measure of RBC marking. Reticulocyte counts dropped from $\sim 34 \%$ (in the SFFV-EGFP group) to $11 \%$, indicative of reduced erythropoietic stress as a consequence of reduced hemolysis and improved RBC survival.

Consistent with reduced rates of erythropoiesis and lower reticulocyte counts, we also observed a striking difference between treatment groups in the frequency of $\mathrm{CD} 71^{+}$Ter $119^{+}$erythroid precursors in the peripheral blood (Figure $5 \mathrm{~B}$ ). In animals that received WT cells, this population was rare $(2 \%)$ and similar to untreated healthy mice. This population of stress-induced immature cells was significantly reduced from $33 \% \pm 5 \%$ to $6 \% \pm 5 \%$ in the LCR-shRNA ${ }^{\text {miR }} 5-$ treated group compared with mice that received SFFV-EGFP-transduced HSCs derived from BERK-SCD.

\section{Discussion}

Here we present preclinical data using an innovative gene therapy approach for the treatment of SCD. In contrast with approaches having the goal of recombinant expression of an antisickling variant of the $\beta$-globin chain, our approach of downregulation of BCL11A exploits the physiological genetic switch that represses $\gamma$-globin expression in a developmental fashion. By effectively knocking down expression of BCL11A, this approach has the important advantage of simultaneously repressing mutant $\beta$-globin expression while inducing protective $\gamma$-globin expression in a balanced fashion. The resulting induction of $\mathrm{HbF}$ at high levels in RBCs would be predicted to markedly attenuate the sickling characteristics of $\mathrm{Hb}^{\mathrm{s} / \mathrm{s}}$ (homozygous for the sickle cell allele) cells. In addition, this approach of $\mathrm{HbF}$ derepression may also be beneficial in $\beta$-thalassemia, a disorder of $\beta$-globin production. Importantly, our data confirm the lack of any negative effect of BCL11A deficiency on RBC development, a key to safe translation of this technology into humans.

As a first step, we developed pol II-driven shRNA ${ }^{\text {miR }}$ s to avoid the previously reported nonspecific toxicity related to shRNA overexpression (21). We discovered an unexpected and profound HSC engraftment defect as a consequence of BCL11A knockdown in both human and mouse repopulating HSCs. This observation, while similar to recent reports in murine $B c l 11 a^{-/}$mice in a normal physiological state (24), extends the mechanistic understanding of the murine HSC phenotype to human engrafting HSCs, indicating its biological relevance. In other transplant models, increased HSC cell cycling has been associated with HSC exhaustion (3). We surmise that this is the cause of reduced engraftment in gene-modified HSCs. Further investigations will be required to fully elucidate the detailed functions and pathways modulated by BCL11A in HSCs and for a better mechanistic understanding beyond the current descriptive work.

By utilizing erythroid-specific pol II-dependent regulatory elements to express shRNA ${ }^{\mathrm{miR}} \mathrm{s}$, the untoward effect of BCL11A knockdown on engrafting HSCs was mitigated. An additional benefit of this vector configuration is the favorable genotoxicity profile reported for similar vectors $(28,29)$. While detailed genotoxity studies are ongoing, the transcriptional fidelity of the LCR vector also reduces the chance of dysregulating cellular genes in HSCs via transactivation or read-through mechanisms.

We observed that LCR-mediated expression of shRNA $^{\mathrm{miR}} \mathrm{S}$ is even more efficient in terms of BCL11A knockdown and $\gamma$-globin induction than expression from the strong and ubiquitously active SFFV promoter. Interestingly, this finding implies that there is no substantial regulation of the LCR vector's cis-regulatory elements mediated by BCL11A, which would result in a negative feedback loop. Epigenetic marks and long-distance contacts between hypersensitive sites within the LCR and the $\gamma$ - or $\beta$-globin promoter precedes active transcription of the locus (30). Since we are able to efficiently reverse the hemoglobin switch in the setting described here, the epigenetic state of the $\beta$-globin cluster does not appear to be irreversibly established by the time of LCR vector-driven BCL11A knockdown.

The top candidate vector LCR-shRNA ${ }^{\text {miR }} 5$ reproducibly mediates greater than $60 \% \mathrm{HbF}$ in both healthy and SCD experimental models, and this vector is currently in large-scale Good Manufacturing Production (GMP) for a planned clinical trial. This level of $\mathrm{HbF}$ exceeds the concentration required for strong antisickling activity seen in patients with hereditary persistence of fetal hemoglobin (HPFH). Individuals with compound HbS-HPFH mutations leading to more than $30 \% \mathrm{HbF}$ have markedly attenuated disease phenotypes (31). However, in contrast with the situation in $\mathrm{HPFH}$, the distribution of $\mathrm{HbF}$ in the gene therapy setting will not be pancellular since a residual fraction of unmodified cells will be present in the engrafted cells. The fraction of uncorrected SCD erythrocytes, and consequently associated complications, will depend on additional critical factors, including the efficiency of transduction of HSCs and the alteration in RBC survival effected by $\gamma$-globin induction (32). In addition, SNPs in the BCL11A gene are strongly associated with higher $\mathrm{HbF}$ levels in the general population (33), which may impact the degree of induction of $\mathrm{HbF}$ after knockdown.

While HSCs transduced with vectors that downregulate BCL11A would be anticipated to have no selective advantage in vivo, an important factor that will strongly augment gene therapy efficacy in this setting is the improved survival of erythrocytes derived from gene-modified HSCs compared with unmodified SCD erythrocytes. Healthy erythrocytes have a 4- to 5-fold longer lifespan compared with SCD erythrocytes. Comparable increases in the half-life and survival have been reported for high-HbF-contain- 
ing SCD cells in the BERK mouse model $(8,15)$. We have utilized these data to create a mathematical model that predicts the estimated fraction of corrected RBCs in peripheral blood as a function of corrected HSCs in the bone marrow (16) and validated the model experimentally. Using this model and published data, an estimated $20 \%$ of the HSCs need to be genetically modified to reduce the fraction of cells expressing high levels of $\mathrm{HbS}$ in $\mathrm{Hb} \beta^{\mathrm{s} / \mathrm{s}}$ cells to less than $30 \%$, which has been shown to be an effective threshold in stroke prevention trials $(34,35)$. This level of gene transfer is attainable using current lentivirus vectors in the clinical setting.

Three different gene therapy trials for SCD have recently been initiated (reviewed in refs. 36 and 37), all based on expression of a chimeric $\beta$-like globin chain with antisickling properties. These trials all utilize lentiviral vectors and incorporate regulatory elements for transgene expression similar to the vector described here. Although differences in experimental procedures exist, our vector appears to compare favorably in in vitro-differentiated erythrocytes derived from transduced CD34 cells when compared with the results published by Romero et al. (38). In that study, the average yield per FB-AS $\beta$ vector copy is $\sim 25 \%$ of total $\beta$-like globin mRNA representing $\sim 18 \% \mathrm{HbF}$ protein, while we obtain in the range of $50 \% \gamma$-globin mRNA and $\mathrm{HbF}$ per vector copy number using the LCR-shRNA ${ }^{\text {miR } 5}$ vector (Figure $3 \mathrm{C}$ ). In addition, and in contrast with the trials currently underway, using the strategy of BCL11A knockdown simultaneously effects a balanced reduction in $\beta^{\text {s }}$, which may have significant therapeutic advantages. Nonetheless, the relative merit of the various approaches is difficult to estimate based on published preclinical and clinical data.

In summary, we have shown an unexpected and severe toxicity of BCL11A knockdown in repopulating HSCs that has direct and important consequences for translation into human gene therapy trials. By utilizing lineage-specific and miRNA-embedded expression of BCL11A-targeting shRNAs, we demonstrate the capacity of lentivirus vectors to effect $\gamma$-globin induction leading to clinically significant increases in $\mathrm{RBC} \mathrm{HbF}$ while obviating this toxicity. While correction of the sickle cell point mutation in HSCs by genome editing is inefficient due to its dependence on homologydirected repair (39), an alternative approach aiming at targeted deletion of BCL11A by gene editing may be achieved at higher efficiencies $(40,41)$. Extrapolation of the data presented here suggests that genetic engineering of the BCL11A coding sequence would likely reduce the long-term engraftment potential of genetically engineered cells and is thus not a viable therapeutic approach. An alternative approach bypassing this problem could be the targeted inactivation of the erythroid-specific enhancer of BCL11A by introducing indels using gene editing technologies $(42,43)$. Although the levels of $\mathrm{HbF}$ induction in vitro appear to be comparable to our approach, the effect of BCL11A enhancer disruption on engrafting HSCs and the level of $\mathrm{HbF}$ induction after engraftment is currently unknown. More generally speaking, assessment of the safety and specificity of gene editing in HSCs and upscaling and manufacturing for clinical applications faces the same challenges as viral vectors did a decade ago, and many aspects of this new technology remain to be explored. Unlike gene editing, the use of viral vectors described here has the advantage that extensive expertise in factors important for clinical translation such as safety, efficacy, and high transduction efficiencies exists from a decade of clinical studies, and protocols scaled and utilized in these clinical trials are directly applicable to the approach described here. Owing to the selective survival advantage of nonsickling RBCs, we predict that this approach would lead to the majority of RBCs being nonsickling in human gene therapy applications, resulting in significant disease attenuation.

\section{Methods}

Construction of shRNA miR constructs. The generation of SFFV-shRNA ${ }^{\text {miR }}$ vectors has been previously described (21). LCR-shRNA ${ }^{\text {miR }}$ vectors were generated by deleting the $\beta$-globin-BGHpA elements of the pRRLHS3-HS2- $\beta$-globin (Globe; see ref. 25 ) vector by digesting with EcoRV and subsequent insertion of an NaeI-digested preassembled VenusshRNA ${ }^{\text {miR }}$-BGHpA cassette. This cassette was generated by ligation of a Venus PCR fragment into PCR 2.1 TOPO (forward, TTGCCGGCATGGTGAGCAAGGGCGAGG; reverse, TTGCCGGCATGGTGAGCAAGGGCGAGG ) followed by insertion of a synthesized shRNA ${ }^{\text {miR }}$ sequence into the NotI-PspOMI-digested plasmid. The PCR-amplified BGHpA (forward, CGCTCGAGCATGCATCTAGAGG; reverse, CGCTCGAGCATGCATCTAGAGG) was introduced into the NotI site of the preexisting elements. SFFV-EGFP and SFFV-BFP vectors without any shRNA cassette were used as controls $(44,45)$.

Virus production and titration. Lentiviral vector supernatants were produced by transfection of $10 \mu \mathrm{g}$ of lentiviral vector, $5 \mu \mathrm{g}$ of gag-pol, $2.5 \mu \mathrm{g}$ of rev and $2.5 \mu \mathrm{g}$ of VSVG packaging plasmids into HEK 293T/17 (ATCC CRL-11268) in 10-cm plates. Plasmids were mixed with 1 $\mathrm{ml} \mathrm{DMEM} \mathrm{(Cellgro)} \mathrm{and} 70 \mu \mathrm{l}$ of $1 \mathrm{mg} / \mathrm{ml}$ linear PEI (Polysciences), incubated for 15-30 minutes, and added to the culture dish. Media exchange was performed 14 hours later and virus supernatants were collected 48 hours after transfection. Virus concentration was performed as described in (21). Infectious titers were determined on mouse erythroid leukemia (MEL) cells by applying serial dilutions of vector supernatant followed by erythroid differentiation for 4 days in RPMI (Cellgro) supplemented with 1.25\% DMSO and 5\% FCS.

Erythroid in vitro differentiation of $\mathrm{CD}_{3} 4^{+}$cells. Transductions, erythroid differentiation, and analyses of $\mathrm{CD} 34^{+}$cells were performed as described (21). Frozen mobilized peripheral blood healthy donor $\mathrm{CD}_{34}{ }^{+}$cells were obtained from the Fred Hutchinson Cancer Research Center (Seattle, Washington, USA), and sickle cell bone marrow $\mathrm{CD}_{3} 4^{+}$cells were isolated from bone marrow aspirates obtained from patients at Boston Children's Hospital after informed consent under a protocol approved by the Institutional Review Board.

Isolation, transduction, and flow cytometric analysis of human CD34+ cells for transplantation experiments. Sickle cell patient bone marrow $\mathrm{CD} 34^{+}$cells were isolated from bone marrow aspirates using the Miltenyi CD34 Microbead kit (Miltenyi Biotec). Cells were prestimulated for 24 hours at a density of $\sim 1 \times 10^{6}$ cells $/ \mathrm{ml}$ in $100 \mathrm{ng} /$ $\mathrm{ml}$ human stem cell factor (hSCF), $40 \mathrm{ng} / \mathrm{ml} \mathrm{hIL-3} \mathrm{(both} \mathrm{Peprotech),}$ $100 \mathrm{ng} / \mathrm{ml} \mathrm{hFlt3-L}$, and $100 \mathrm{ng} / \mathrm{ml} \mathrm{hTPO}$ in CellGro GMP medium (both Cellgenix) followed by transduction. Media exchange was performed after an additional 14-16 hours and cells were allowed to recover overnight before transplantation into conditioned (2.7 Gy) NSG mice (NOD/LtSz-scid Il2 $\mathrm{rg}^{-/-}$) at a dose of 0.5-2.5 $\times 10^{6}$ cells per recipient. For flow cytometric analyses the following antibodies were used: hCD45-PE (clone 5B1), mCD45 (clone 30F11), mouse and human FcR-block (catalog 130-059-901 and 130-092-575) (all from Miltenyi). hCD34 (clone 581), hCD3 (clone UCHT1), hCD19 (clone 
SJ25C1), hCD71 (clone M-A712) and GpA (clone GA-R2 (HIR2)) (all from BD Pharmingen) and eFluor 780 viability dye (catalog 65-086514; eBiosciences).

Isolation, transduction, and flow cytometric analysis of mouse cells for transplantation experiments. Lineage-negative mouse bone marrow cells were isolated by flushing femurs, tibias, and hips of CD45.1 BoyJ (B6.SJL-Ptprca Pepcb/BoyJ) and CD45.2 B6 mice (C57BL/6J) followed by lineage depletion using the Mouse Lineage Cell Depletion Kit (Miltenyi Biotec). Cells were cultured at a density of $\sim 1 \times 10^{6}$ cells $/ \mathrm{ml}$ in the medium described above for $\mathrm{CD} 34^{+}$cells. Following a 24 -hour prestimulation, cells were transduced at a density of $1 \times 10^{6}$ cells $/ \mathrm{ml}$ at an MOI of 40 and transplanted into lethally irradiated ( $7+4 \mathrm{~Gy}$, split dose) recipients 3 days after isolation. For competitive repopulation experiments, equal numbers of cells from different transduction groups were mixed prior to transplantation into CD45.2 or heterozygous CD45.1/ CD45.2 double-positive recipients $\left(0.4-1 \times 10^{6}\right.$ cells per animal). Cell mixtures were analyzed via flow cytometry to confirm equal contributions of both competitor cell fractions, and readjusted if required. Analysis of peripheral blood, bone marrow, and spleens was performed at multiple timepoints using the following antibodies: CD45.1 (clone A20; Biolegend), CD45.2 (clone 104-2; Miltenyi), B220 (clone RA3-6B2; BD Pharmingen), CD11b (clone M1/70; Miltenyi), CD3 (clone 145-2C11; Biolegend), CD71 (clone C2; BD Pharmingen), Ter119 (clone Ter-119; BD Pharmingen), and fixable viability dye eFluor 780. For analysis of the erythroid lineage, RBC lysis was omitted.

Apoptosis and cell cycle stain. The viability of CD $34^{+}$cells was assessed 48 hours after transduction using the Dead Cell Apoptosis Kit with APC annexin V (Molecular Probes) and 7-aminoactinomycin D according to the manufacturer's instructions. Alternatively, the cell cycle status was assessed by using the Cytofix/Cytoperm Kit (BD Biosciences) followed by staining with Ki67-PE-Cy7 (BD Pharmingen) and DAPI.

Flow cytometric analyses and cell sorting. Analyses were performed on an LSR-II or LSRFortessa flow cytometer (Becton Dickinson) and Diva or FloJoX (Treestar) software. Data analyses and statistics were done using Excel (Microsoft) and Graphpad Prism 5. Cell sorting was performed on a FACSAria II machine (Becton Dickinson).

Additional procedures and material. RNA extraction, reverse transcription, qPCR, Western blots, and HPLC were performed as described in (21).

Statistics. Data was analyzed by 2-tailed $t$ test or one-way ANOVA and Bonferroni's multiple comparisons post test (95\% confidence interval) using Graphpad Prism software. ${ }^{*} P \leq 0.05,{ }^{* *} P \leq 0.01$, and ${ }^{* * *} P \leq 0.001$ were considered significant.

\section{Author contributions}

$\mathrm{CB}, \mathrm{SG}, \mathrm{RR}, \mathrm{DB}, \mathrm{MC}, \mathrm{DK}, \mathrm{JF}$, and MM all performed experimental work. YJK and MH provided patient samples and provided technical assistance. CB, SHO, RG, and DAW wrote and edited the paper.

\section{Acknowledgments}

We thank Chad Harris, Meaghan McGuinness, Carlo Brugnara, and the Clinical and Translational Investigation Program and the TransLab at BCH for technical assistance; and Giuliana Ferrari, Luigi Naldini, and Bernhard Gentner for plasmids. This work was supported in part by NIH grants HL117720-03 (to SHO, CB, and DAW) and DK093705 (to DEB), Doris Duke Charitable Foundation Innovations in Clinical Research Award grant 2013137 (to DEB), and sponsored research support from Bluebird Bio (to DAW and SG). We also thank Maria Suarez and Natasha Rossi for administrative assistance.

Address correspondence to: David A. Williams, 300 Longwood Avenue, Karp 08125.3, Boston, Massachusetts 02115, USA. Phone: 617.919.2697; E-mail: DAWilliams@childrens.harvard.edu.

R. Renella's present address is: Département Médico-Chirurgical de Pédiatrie, Centre Hospitalier Universitaire Vaudois, Lausanne, Switzerland.
1. Musallam KM, Sankaran VG, Cappellini MD, Duca L, Nathan DG, Taher AT. Fetal hemoglobin levels and morbidity in untransfused patients with $\beta$-thalassemia intermedia. Blood. 2012;119(2):364-367.

2. Sankaran VG, et al. Human fetal hemoglobin expression is regulated by the developmental stage-specific repressor BCL11A. Science. 2008;322(5909):1839-1842.

3. Xu J, et al. Transcriptional silencing of \{gamma\}-globin by BCL11A involves long-range interactions and cooperation with SOX6. Genes Dev. 2010;24(8):783-798.

4. Xu J, et al. Corepressor-dependent silencing of fetal hemoglobin expression by BCL11A. Proc Natl Acad Sci U S A. 2013;110(16):6518-6523.

5. Sankaran VG, et al. Developmental and species-divergent globin switching are driven by BCL11A. Nature. 2009;460(7259):1093-1097.

6. Liu P, et al. Bcl11a is essential for normal lymphoid development. Nat Immunol. 2003;4(6):525-532.

7. Yu Y, et al. Bcl11a is essential for lymphoid development and negatively regulates p53. J Exp Med. 2012;209(13):2467-2483.
8. $\mathrm{Xu}$ J, et al. Correction of sickle cell disease in adult mice by interference with fetal hemoglobin silencing. Science. 2011;334(6058):993-996.

9. Walters MC, et al. Impact of bone marrow transplantation for symptomatic sickle cell disease: an interim report. Multicenter investigation of bone marrow transplantation for sickle cell disease. Blood. 2000;95(6):1918-1924.

10. Hansbury EN, Schultz WH, Ware RE, Aygun B. Bone marrow transplant options and preferences in a sickle cell anemia cohort on chronic transfusions. Pediatr Blood Cancer. 2012;58(4):611-615.

11. Walters MC, et al. Stable mixed hematopoietic chimerism after bone marrow transplantation for sickle cell anemia. Biol Blood Marrow Transplant. 2001;7(12):665-673.

12. Pawliuk R, et al. Correction of sickle cell disease in transgenic mouse models by gene therapy. Science. 2001;294(5550):2368-2371.

13. Levasseur DN, Ryan TM, Reilly MP, McCune SL, Asakura T, Townes TM. A recombinant human hemoglobin with anti-sickling properties greater than fetal hemoglobin. J Biol Chem. 2004;279(26):27518-27524.

14. Pestina TI, Hargrove PW, Jay D, Gray JT, Boyd
KM, Persons DA. Correction of murine sickle cell disease using gamma-globin lentiviral vectors to mediate high-level expression of fetal hemoglobin. Mol Ther. 2009;17(2):245-252.

15. Perumbeti A, et al. A novel human gamma-globin gene vector for genetic correction of sickle cell anemia in a humanized sickle mouse model: critical determinants for successful correction. Blood. 2009;114(6):1174-1185.

16. Altrock PM, Brendel C, Renella R, Orkin SH, Williams DA, Michor F. Mathematical modeling of erythrocyte chimerism informs genetic intervention strategies for sickle cell disease [published online ahead of print June 14, 2016]. Am J Hematol. doi: 10.1002/ajh.24449.

17. Williams DA, Thrasher AJ. Concise review: lessons learned from clinical trials of gene therapy in monogenic immunodeficiency diseases. Stem Cells Transl Med. 2014;3(5):636-642.

18. Williams DA. Broadening the indications for hematopoietic stem cell genetic therapies. Cell Stem Cell. 2013;13(3):263-264.

19. Grimm D, et al. Argonaute proteins are key determinants of RNAi efficacy, toxicity, and persistence in the adult mouse liver. J Clin Invest. 
2010;120(9):3106-3119.

20. Martin JN, Wolken N, Brown T, Dauer WT, Ehrlich ME, Gonzalez-Alegre P. Lethal toxicity caused by expression of shRNA in the mouse striatum: implications for therapeutic design. Gene Ther. 2011;18(7):666-673.

21. Guda S, et al. miRNA-embedded shRNAs for lineage-specific BCL11A knockdown and hemoglobin F induction. Mol Ther. 2015;23(9):1465-1474.

22. Amendola M, Passerini L, Pucci F, Gentner B, Bacchetta R, Naldini L. Regulated and multiple miRNA and siRNA delivery into primary cells by a lentiviral platform. Mol Ther. 2009;17(6):1039-1052.

23. Weber K, Bartsch U, Stocking C, Fehse B. A multicolor panel of novel lentiviral "gene ontology" (LeGO) vectors for functional gene analysis. $\mathrm{Mol}$ Ther. 2008;16(4):698-706.

24. Tsang JC, et al. Single-cell transcriptomic reconstruction reveals cell cycle and multi-lineage differentiation defects in Bcl11a-deficient hematopoietic stem cells. Genome Biol. 2015;16:178.

25. Miccio A, et al. In vivo selection of genetically modified erythroblastic progenitors leads to long-term correction of beta-thalassemia. Proc Natl Acad Sci U S A. 2008;105(30):10547-10552.

26. Funnell AP, et al. 2p15-p16.1 microdeletions encompassing and proximal to BCL11A are associated with elevated $\mathrm{HbF}$ in addition to neurologic impairment. Blood. 2015;126(1):89-93.

27. Basak A, et al. BCL11A deletions result in fetal hemoglobin persistence and neurodevelopmental alterations. J Clin Invest. 2015;125(6):2363-2368.

28. Roselli EA, et al. Correction of beta-thalassemia major by gene transfer in haematopoietic pro- genitors of pediatric patients. EMBO Mol Med. 2010;2(8):315-328.

29. Arumugam PI, et al. Genotoxic potential of lineage-specific lentivirus vectors carrying the beta-globin locus control region. Mol Ther. 2009;17(11):1929-1937.

30. Palstra RJ, Tolhuis B, Splinter E, Nijmeijer R, Grosveld F, de Laat W. The beta-globin nuclear compartment in development and erythroid differentiation. Nat Genet. 2003;35(2):190-194.

31. Ngo DA, et al. Fetal haemoglobin levels and haematological characteristics of compound heterozygotes for haemoglobin $\mathrm{S}$ and deletional hereditary persistence of fetal haemoglobin. $\mathrm{Br} \mathrm{J}$ Haematol. 2012;156(2):259-264.

32. Steinberg MH, Chui DH, Dover GJ, Sebastiani P, Alsultan A. Fetal hemoglobin in sickle cell anemia: a glass half full? Blood. 2014;123(4):481-485.

33. Uda M, et al. Genome-wide association study shows BCL11A associated with persistent fetal hemoglobin and amelioration of the phenotype of beta-thalassemia. Proc Natl Acad Sci U S A. 2008;105(5):1620-1625.

34. Aygun B, et al. Chronic transfusion practice for children with sickle cell anaemia and stroke. $\mathrm{Br} \mathrm{J}$ Haematol. 2009;145(4):524-528.

35. Ware RE, et al. TCD with transfusions changing to hydroxyurea (TWiTCH): hydroxyurea therapy as an alternative to transfusions for primary stroke prevention in children with sickle cell anemia. Blood. 2015;126(23):3.

36. Hoban MD, Orkin SH, Bauer DE. Genetic treatment of a molecular disorder: gene therapy approaches to sickle cell disease. Blood. 2016;127(7):839-848.
37. Negre O, et al. Gene therapy of the $\beta$-hemoglobinopathies by lentiviral transfer of the $\beta(\mathrm{A}(\mathrm{T} 87 \mathrm{Q}))$-globin gene. Hum Gene Ther. 2016;27(2):148-165.

38. Romero $Z$, et al. $\beta$-globin gene transfer to human bone marrow for sickle cell disease. J Clin Invest. 2013;123(8):3317-3330.

39. Hoban MD, et al. Correction of the sickle cell disease mutation in human hematopoietic stem/ progenitor cells. Blood.2015;125(17):2597-2604.

40. Urnov FD, et al. Clinical-scale genome editing of the human BCL11A erythroid enhancer for treatment of the hemoglobinopathies. Blood. 2015;126(23):204.

41. Chang $\mathrm{KH}$, et al. Clonal analysis of human bone marrow CD $34^{+}$cells edited by BCL11A-targeting zinc finger nucleases reveals clinically relevant levels of fetal globin expression in edited erythroid progeny. Blood. 2015;126(23):3234.

42. Bauer DE, et al. An erythroid enhancer of BCL11A subject to genetic variation determines fetal hemoglobin level. Science. 2013;342(6155):253-257.

43. Canver MC, et al. BCL11A enhancer dissection by Cas9-mediated in situ saturating mutagenesis. Nature. 2015;527(7577):192-197.

44. Demaison C, et al. High-level transduction and gene expression in hematopoietic repopulating cells using a human immunodeficiency [correction of imunodeficiency] virus type 1-based lentiviral vector containing an internal spleen focus forming virus promoter. Hum Gene Ther. 2002;13(7):803-813.

45. Brendel C, et al. CD133-targeted gene transfer into long-term repopulating hematopoietic stem cells. Mol Ther. 2015;23(1):63-70. 